Article

\title{
A Unified Convergence Analysis for Some Two-Point Type Methods for Nonsmooth Operators
}

\author{
Sergio Amat ${ }^{1, *, \dagger}$, Ioannis Argyros ${ }^{2, \dagger}$, Sonia Busquier ${ }^{1, \dagger}$, Miguel Ángel Hernández-Verón ${ }^{3,+} \mathbb{C}$ \\ and María Jesús Rubio ${ }^{3,+}$ \\ 1 Departamentode Matemática Aplicada y Estadística, Universidad Politécnica de Cartagena, \\ 11003 Cádiz, Spain \\ 2 Department of Mathematical Sciences, Cameron University, Lawton, OK 73505, USA \\ 3 Departamento de Matemáticas y Computación, Universidad de La Rioja, Calle Madre de Dios, 53, \\ 26006 Logrono, Spain \\ * Correspondence: sergio.amat@upct.es; Tel.: +34-968-325-651 \\ + These authors contributed equally to this work.
}

Received: 18 June 2019; Accepted: 31 July 2019; Published: 3 August 2019

\begin{abstract}
The aim of this paper is the approximation of nonlinear equations using iterative methods. We present a unified convergence analysis for some two-point type methods. This way we compare specializations of our method using not necessarily the same convergence criteria. We consider both semilocal and local analysis. In the first one, the hypotheses are imposed on the initial guess and in the second on the solution. The results can be applied for smooth and nonsmooth operators.
\end{abstract}

Keywords: iterative methods; nonlinear equations; Newton-type methods; smooth and nonsmooth operators

\section{Introduction}

One of the most important techniques in order to approximate nonlinear equations are iterative methods [1-6]. In this paper, we present a unified approach for two-point Newton-type methods for smooth and nonsmooth operators [7-10]. We will consider two types of convergence. The semilocal convergence is where the hypotheses are imposed on the initial guess; and local convergence is where the hypotheses are imposed on the solution. Our family includes a great variety of methods. We are interested also in the application of these methods in practice (nonlinear systems, boundary problems and image processing).

For a greater generality, in this study, let $X$ and $Y$ be two Banach spaces and $D$ a nonempty, open, and convex set; let $F_{1}: D \subset X \rightarrow Y$ and $F_{2}: D \subset X \rightarrow Y$ be continuous operators. Moreover, we assume that the operator $F_{1}$ has a continuous Fréchet derivative and $F_{2}$ is a continuous operator whose differentiability is not assumed. We consider the equation

$$
F(x)=F_{1}(x)+F_{2}(x)=0 .
$$

To solve this equation, we use the two-point Newton-type methods defined by

$$
x_{k+1}=x_{k}-L_{k-1, k}^{-1}\left(F_{1}\left(x_{k}\right)+F_{2}\left(x_{k}\right)\right)
$$

for each $k=0,1,2, \ldots$, where $x_{-1}, x_{0} \in D$ are the initial points, $L(.,):. D \times D \rightarrow \mathfrak{L}(X, Y)$ and $\mathfrak{L}(X, Y)$ is the space of bounded linear operators from $X$ into $Y$. We have denoted by $L_{k-1, k}=L\left(x_{k-1}, x_{k}\right)$.

If $F_{2}(x) \neq 0$, we have that the operator $F$ is not Fréchet differentiable. In general, to approximate a solution of (1) in this situation, derivative-free iterative methods are used [11-14]. To obtain this 
type of iterative processes, it is common to approximate derivatives by difference divided. Remember that, given an operator $H: D \subset X \rightarrow Y$, we call $[x, y ; H] \in \mathfrak{L}(X, Y)$ a first order divided differences operator for $H$ on the points $x$ and $y(x \neq y)$ in $D$ if

$$
[x, y ; H](x-y)=H(x)-H(y) .
$$

So, to solve (1) with iterative methods given from (2), we can consider at least two different procedures. Firstly, we have the Zincenko method [15], given by the following algorithm:

$$
\left\{\begin{array}{l}
\text { Given } x_{-1}, x_{0} \in D, \\
x_{k+1}=x_{k}-\left[F_{1}^{\prime}\left(x_{k}\right)\right]^{-1} F\left(x_{k}\right), \quad n \geq 0,
\end{array}\right.
$$

where we directly eliminate the nondifferentiable part of $F$, i.e., $F_{2}$. So, in this case, $L_{k-1, k}=F_{1}^{\prime}\left(x_{k}\right)$ in (2). Secondly, we can consider an approximation of $F^{\prime}$ by divided differences, the secant-type methods $[16,17]$ :

$$
\left\{\begin{array}{l}
\text { Given } x_{-1}, x_{0} \in D, \\
y_{k}=\lambda x_{k}+(1-\lambda) x_{k-1}, \quad \lambda \in[0,1), \\
x_{k+1}=x_{k}-\left[y_{k}, x_{k} ; F\right]^{-1} F\left(x_{k}\right), \quad n \geq 0,
\end{array}\right.
$$

where the secant method, for $\lambda=0$, is obtained. So, in this case, $L_{k-1, k}=\left[y_{k}, x_{k} ; F\right]$ in (2). But, if we consider a better approximation of the derivative of $F$, an approximation of second order, we have the Kurchatov method [18]:

$$
\left\{\begin{array}{l}
\text { Given } x_{-1}, x_{0} \in D, \\
x_{k+1}=x_{k}-\left[x_{k-1}, 2 x_{k}-x_{k-1} ; F\right]^{-1} F\left(x_{n}\right), \quad n \geq 0,
\end{array}\right.
$$

in this case, $L_{k-1, k}=\left[x_{k-1}, 2 x_{k}-x_{k-1} ; F\right]$ in (2).

By using this procedure of decomposition for operator $F$, we see that we can also consider the application of iterative methods that use derivatives when $F$ is nondifferentiable. So, if we consider decomposition of $F$ given in (1), we can use the Newton-secant-type algorithm:

$$
\left\{\begin{array}{l}
\text { Given } x_{-1}, x_{0} \in D, \\
y_{k}=\lambda x_{k}+(1-\lambda) x_{k-1}, \quad \lambda \in[0,1), \\
x_{k+1}=x_{k}-\left(F_{1}^{\prime}\left(x_{k}\right)+\left[y_{k}, x_{k} ; F\right]\right)^{-1} F\left(x_{k}\right), \quad n \geq 0,
\end{array}\right.
$$

where $L_{k-1, k}=F_{1}^{\prime}\left(x_{k}\right)+\left[y_{k}, x_{k} ; F\right]$ in (2). The other possibility, from the decomposition method, is to consider the Newton-Kurchatov [19] algorithm:

$$
\left\{\begin{array}{l}
\text { Given } x_{-1}, x_{0} \in D, \\
x_{k+1}=x_{k}-\left(F_{1}^{\prime}\left(x_{k}\right)+\left[x_{k-1}, 2 x_{k}-x_{k-1} ; F\right]\right)^{-1} F\left(x_{k}\right), \quad n \geq 0,
\end{array}\right.
$$

where $L_{k-1, k}=F_{1}^{\prime}\left(x_{k}\right)+\left[x_{k-1}, 2 x_{k}-x_{k-1} ; F\right]$ in (2). Another possibility is to consider Steffensen-type methods, that is, the methods associated to divided differences like $\left[x_{k}, x_{k}+F\left(x_{k}\right) ; F\right]$.

As we can see, there are a lot of iterative methods that can be written as algorithms (2).

The main aim of this paper is to obtain a general study for the convergence, local and semilocal, for these Newton-type of iterative methods given in (2). 


\section{Convergence Analysis for Two-Point Newton-Type Methods}

In this section, we present both semilocal and local convergence analysis. In the first one, the hypotheses are imposed on the initial guess; and in the second, on the solution. The results can be applied for smooth and nonsmooth operators.

\subsection{Local Convergence Analysis}

We start the local analysis of method (2). Let $v_{0}:[0,+\infty) \times[0,+\infty) \rightarrow[0,+\infty)$ be a nondecreasing continuous function. Assume that the equation

$$
v_{0}(t, t)=1
$$

has at least one positive root $r_{0}$. Let also $v:\left[0, r_{0}\right) \times\left[0, r_{0}\right) \rightarrow[0,+\infty)$ be a nondecreasing continuous function. Define function $\bar{v}$ on the interval $\left[0, r_{0}\right)$ by

$$
\bar{v}(t)=\frac{v(t, t)}{1-v_{0}(t, t)}-1
$$

Assume that the equation

$$
\bar{v}(t)=0
$$

has a minimal positive solution $r$. It follows that for each $t \in[0, r)$

$$
0 \leq v_{0}(t, t)<1
$$

and

$$
0 \leq \bar{v}(t)<1
$$

Our analysis of method (2) will use the conditions (A):

- $\quad\left(a_{1}\right)$ There exist a solution $x^{*} \in D$ of Equation (1), and $B \in \mathfrak{L}(X, Y)$ such that $B^{-1} \in \mathfrak{L}(Y, X)$.

- $\quad\left(a_{2}\right)$ Condition (9) holds and for each $x, u \in D$

$$
\left\|B^{-1}(L(x, u)-B)\right\| \leq v_{0}\left(\left\|x-x^{*}\right\|,\left\|u-y^{*}\right\|\right),
$$

where $v_{0}$ is defined previously, and $r_{0}$ is given in (9).

Set $D_{0}=D \cap \bar{U}\left(x^{*}, r_{0}\right)$.

- $\quad\left(a_{3}\right)$ For each $x, z \in D_{0}$, and any solution $y \in D$ of Equation (1)

$$
\left\|B^{-1}\left(F_{1}(x)+F_{2}(x)-L(z, x) y\right)\right\| \leq v(\|z-y\|,\|x-y\|)\|x-y\|,
$$

where $v$ is defined previously, and $L(\cdot, \cdot): D_{0} \times D_{0} \rightarrow \mathfrak{L}(X, Y)$.

- $\quad\left(a_{4}\right) \bar{U}\left(x^{*}, r\right) \subset D$, where $r$ is given in (10).

- $\left(a_{5}\right)$

$$
\xi:=\frac{v(r, r)}{1-v_{0}(r, r)} \in[0,1)
$$

We are able to perform our local analysis of method (2) based on the aformentioned conditions (A).

Theorem 1. Assume that the conditions (A) hold. Then, sequence $x_{k}$, defined by method (2) for $x_{-1}, x_{0} \in$ $U\left(x^{*}, r\right)-x^{*}$, is well defined in $U\left(x^{*}, r\right)$; remains in $U\left(x^{*}, r\right)$; and converges to $x^{*}$. Finally, the following estimates hold.

$$
\left\|x_{k+1}-x^{*}\right\| \leq \frac{v\left(\left\|x_{k-1}-x^{*}\right\|,\left\|x_{k}-x^{*}\right\|\right)}{1-v_{0}\left(\left\|x_{k-1}-x^{*}\right\|,\left\|x_{k}-x^{*}\right\|\right)}\left\|x_{k}-x^{*}\right\| \leq\left\|x_{k}-x^{*}\right\|<r .
$$


The vector $x^{*}$ is the only solution of Equation (1) in $\bar{U}\left(x^{*}, r\right)$.

Proof. We will use mathematical induction on $k$.

Let $x, u \in U\left(x^{*}, r\right)$.

Using (2), $\left(a_{1}\right)$ and $\left(a_{2}\right)$, we obtain

$$
\left\|B^{-1}(L(x, u)-B)\right\| \leq v_{0}\left(\left\|x-x^{*}\right\|,\left\|u-x^{*}\right\|\right) \leq v_{0}(r, r)<1 .
$$

Using the Banach lemma on invertible operators [20] and (15), we deduce that $L(x, u)^{-1} \in$ $\mathfrak{L}(Y, X)$, and

$$
\left\|L(x, u)^{-1} B\right\| \leq \frac{1}{1-v_{0}\left(\left\|x-x^{*}\right\|,\left\|u-x^{*}\right\|\right)} .
$$

In particular, estimate (16) holds for $x=x_{0}$, so $x_{1}$ is well defined by method (2) for $k=0$.

Using the definition of the method (2) (for $k=0) ;\left(a_{1}\right),\left(a_{3}\right),(13)$, and (16) (for $\left.k=0\right)$ that

$$
\begin{aligned}
\left\|x_{1}-x^{*}\right\| & =\left\|x_{0}-x^{*}-L\left(x_{-1}, x_{0}\right)^{-1}\left(x_{-1}, x_{0}\right)\left(F_{1}\left(x_{0}\right)+F_{2}\left(x_{0}\right)\right)\right\| \\
& =\left\|\left[L\left(x_{-1}, x_{0}\right)^{-1} B\right]\left[B^{-1}\left(F_{1}\left(x_{0}\right)+F_{2}\left(x_{0}\right)-L\left(x_{-1}, x_{0}\right)\left(x_{0}-x^{*}\right)\right)\right]\right\| \\
& \leq\left\|L\left(x_{-1}, x_{0}\right)^{-1} B\right\|\left\|B^{-1}\left(F_{1}\left(x_{0}\right)+F_{2}\left(x_{0}\right)-L\left(x_{-1}, x_{0}\right)\left(x_{0}-x^{*}\right)\right)\right\| \\
& \leq \frac{v\left(\left\|x_{-1}-x^{*}\right\|,\left\|x_{0}-x^{*}\right\|\right)}{1-v_{0}\left(\left\|x_{-1}-x^{*}\right\|,\left\|x_{0}-x^{*}\right\|\right)}\left\|x_{0}-x^{*}\right\| \leq\left\|x_{0}-x^{*}\right\|<r,
\end{aligned}
$$

which shows estimate (14) for $k=0$ and $x_{1} \in U\left(x^{*}, r\right)$.

Replace $x_{0}, x_{1}$ by $x_{i}, x_{i+1}$ in the preceding estimates to complete the induction for estimate (14). Then, from the estimate

$$
\left\|x_{i+1}-x^{*}\right\| \leq \mu\left\|x_{i}-x^{*}\right\|<r
$$

where

$$
\mu=\frac{v\left(\left\|x_{-1}-x\right\|,\left\|x_{0}-x^{*}\right\|\right)}{1-v_{0}\left(\left\|x_{-1}-x^{*}\right\|,\left\|x_{0}-x^{*}\right\|\right)} \in[0,1),
$$

thus, $\lim _{i \rightarrow+\infty} x_{i}=x^{*}$ and $x_{i+1} \in U\left(x^{*}, r\right)$. Moreover, for the uniqueness part, let $y^{*} \in \bar{U}\left(x^{*}, r\right)$ with $F_{1}\left(y^{*}\right)+F_{2}\left(y^{*}\right)=0$. Using $(a 3),(a 5)$, and estimate (17), we obtain in turn that

$$
\begin{aligned}
\left\|x_{i+1}-y^{*}\right\| & \leq\left\|L\left(x_{i-1}, x_{i}\right)^{-1} B\right\|\left\|B^{-1}\left(F_{1}\left(x_{i}\right)+F_{2}\left(x_{i}\right)-L\left(x_{i-1}, x_{i}\right)\left(x_{i}-y^{*}\right)\right)\right\| \\
& \leq \frac{v\left(\left\|x_{i-1}-y^{*}\right\|,\left\|x_{i}-y^{*}\right\|\right)}{1-v_{0}\left(\left\|x_{i-1}-y^{*}\right\|,\left\|x_{i}-y^{*}\right\|\right)}\left\|x_{i}-y^{*}\right\| \\
& \leq \mu\left\|x_{i}-y^{*}\right\|<\mu^{i+1}\left\|x_{0}-y^{*}\right\|,
\end{aligned}
$$

which shows $\lim _{i \rightarrow+\infty} x_{i}=y^{*}$-but, we showed $\lim _{i \rightarrow+\infty} x_{i}=x^{*}$. Hence, we conclude that $x^{*}=$ $y^{*}$.

Remark 1. - Condition $\left(a_{3}\right)$ can be replaced by the stronger: for each $x, y \in D_{0}$

$$
\left\|B^{-1}\left(F_{1}(x)+F_{2}(x)-L(x)(x-y)\right)\right\| \leq v_{1}(\|x-y\|)\|x-y\|,
$$

where function $v_{1}$ is as $v$. However, for each $t, t^{\prime} \geq 0$

$$
v\left(t, t^{\prime}\right) \leq v_{1}\left(t, t^{\prime}\right)
$$

- Linear operator $B$ does not necessarily depend on $q$, where $q=x^{*}$ or $q=x_{0}$. It is used to determine the invertibility of linear operator $L(\cdot, \cdot)$ appearing in the method. The invertibility of $B$ can be assured by 
an additional condition of the form $\|I-B\|<1$ or in some other way. A possible choice for $B$ is $B=B(q)$ or $B=F_{1}^{\prime}(q)$.

- It follows from the definition of $r_{0}$ and $r$ that $r_{0} \geq r$.

\subsection{Semilocal Convergence Analysis}

For the semilocal case, we also define some functions and parameters. Let $w_{0}:[0,+\infty) \times$ $[0,+\infty) \rightarrow[0,+\infty)$ be a continuous and nondecreasing function.

Assume that the equation

$$
w_{0}(t, t)=1,
$$

has one smallest positive root that we denote by $\rho_{0}$. Let $w:\left[0, \rho_{0}\right) \times\left[0, \rho_{0}\right) \times\left[0, \rho_{0}\right) \rightarrow[0,+\infty)$ be a nondecreasing continuous function. Moreover, for $\eta, \bar{\eta} \geq 0$, define parameters $C_{1}$ and $C_{2}$ by

$$
\begin{aligned}
& C_{1}=\frac{w(\bar{\eta}, \eta, 0)}{1-w_{0}(0, \eta)} \\
& C_{2}=\frac{w\left(0, \frac{\eta}{1-C_{1}}, \eta\right)}{1-w_{0}\left(\eta, \frac{\eta}{1-C_{1}}\right)}
\end{aligned}
$$

and function $C:\left[0, \rho_{0}\right) \rightarrow[0,+\infty)$ by $C(t)=\frac{w(t, t, t)}{1-w_{0}(t, t)}$. Assume that the equation

$$
\left(\frac{C_{1} C_{2}}{1-C(t)}+C_{1}+1\right) \eta-t=0
$$

has one smallest positive root that we denote by $\rho$.

The semilocal convergence analysis of method (2) will be based on conditions $(\mathrm{H})$ :

- (h1) There exists $x_{-1}, x_{0} \in D$, and $B \in \mathfrak{L}(X, Y)$ such that $B^{-1} \in \mathfrak{L}(Y, X)$.

- (h2) Condition (20) holds, and for each $x \in D$

$$
\left\|B^{-1}(L(z, x)-B)\right\| \leq w_{0}\left(\left\|z-x_{0}\right\|,\left\|x-x_{0}\right\|\right),
$$

where $w_{0}$ is defined previously and $\rho_{0}$ is given in (20).

Set $D_{1}=D \cap \bar{U}\left(x_{0}, \rho_{0}\right)$.

- $\quad$ (h3) For $L(\cdot, \cdot): D_{1} \times D_{1} \rightarrow \mathfrak{L}(X, Y)$, and each $x, y, z \in D_{1}$

$$
\begin{array}{r}
\left\|B^{-1}\left(F_{1}(y)-F_{1}(x)+F_{2}(y)-F_{2}(x)-L(z, x)(y-x)\right)\right\| \\
\leq w\left(\left\|z-x_{0}\right\|,\left\|y-x_{0}\right\|,\left\|x-x_{0}\right\|\right)\|y-x\|,
\end{array}
$$

where $w$ is defined previously.

- (h4) $\bar{U}\left(x_{0}, \rho\right) \subseteq D$ and condition (21) holds for $\rho$, where $\left\|x_{1}-x_{0}\right\| \leq \eta$ and $\left\|x_{-1}-x_{0}\right\| \leq \bar{\eta}$. 
Then, using the hypotheses $(\mathrm{H})$, we obtain the estimates:

$$
\begin{aligned}
\left\|x_{2}-x_{1}\right\| & \leq \frac{w\left(\left\|x_{-1}-x_{0}\right\|,\left\|x_{1}-x_{0}\right\|,\left\|x_{0}-x_{0}\right\|\right)\left\|x_{1}-x_{0}\right\|}{1-w_{0}\left(\left\|x_{0}-x_{0}\right\|,\left\|x_{1}-x_{0}\right\|\right)}=C_{1}\left\|x_{1}-x_{0}\right\|, \\
\left\|x_{2}-x_{0}\right\| & \leq\left\|x_{2}-x_{1}\right\|+\left\|x_{1}-x_{0}\right\| \leq\left(1+C_{1}\right)\left\|x_{1}-x_{0}\right\| \\
& =\frac{1-C_{1}^{2}}{1-C_{1}}\left\|x_{1}-x_{0}\right\| \\
& <\frac{\eta}{1-C_{1}}<\rho, \\
\left\|x_{3}-x_{2}\right\| & \leq \frac{w\left(\left\|x_{0}-x_{0}\right\|,\left\|x_{2}-x_{0}\right\|,\left\|x_{1}-x_{0}\right\|\right)}{1-w_{0}\left(\left\|x_{1}-x_{0}\right\|,\left\|x_{2}-x_{0}\right\|\right)}\left\|x_{2}-x_{1}\right\| \\
& \leq \frac{w\left(0, \frac{\eta}{1-C_{1}}, \eta\right)}{1-w_{0}\left(\eta, \frac{\eta}{1-C_{1}}\right)}\left\|x_{2}-x_{1}\right\|=C_{2}\left\|x_{2}-x_{1}\right\|, \\
\left\|x_{3}-x_{0}\right\| & \leq\left\|x_{3}-x_{2}\right\|+\left\|x_{2}-x_{1}\right\|+\left\|x_{1}-x_{0}\right\| \\
& \leq C x_{2}\left\|x_{2}-x_{1}\right\|+C_{1}\left\|x_{1}-x_{0}\right\|+\left\|x_{1}-x_{0}\right\| \\
& \leq\left(C_{2} C_{1}+C_{1}+1\right)\left\|x_{1}-x_{0}\right\|, \\
& \leq \frac{w\left(\left\|x_{1}-x_{0}\right\|,\left\|x_{3}-x_{0}\right\|,\left\|x_{2}-x_{0}\right\|\right)}{1-w_{0}\left(\left\|x_{2}-x_{0}\right\|,\left\|x_{3}-x_{0}\right\|\right)}\left\|x_{3}-x_{2}\right\| \\
& \leq C(\rho)\left\|x_{3}-x_{2}\right\| \leq C(\rho) C_{2}\left\|x_{2}-x_{1}\right\| \\
& \leq C(\rho) C_{2} C_{1}\left\|x_{1}-x_{0}\right\|,
\end{aligned}
$$

similarly for $i=3,4, \ldots$

$$
\begin{array}{rl}
\left\|x_{i+1}-x_{i}\right\| \leq & C(\rho)\left\|x_{i}-x_{i-1}\right\| \leq C(\rho)^{i-2}\left\|x_{3}-x_{2}\right\|, \\
\left\|x_{i+1}-x_{0}\right\| \leq & \left\|x_{i+1}-x_{i}\right\|+\ldots+\left\|x_{4}-x_{3}\right\|+\left\|x_{3}-x_{0}\right\| \\
\leq & C(\rho)\left\|x_{i}-x_{i-1}\right\|+\ldots+C(\rho)\left\|x_{3}-x_{2}\right\| \\
& +\left(C_{2} C_{1}+C_{1}+1\right)\left\|x_{1}-x_{0}\right\| \\
\leq & C(\rho)^{i-2}\left\|x_{3}-x_{2}\right\|+\ldots+C(\rho)\left\|x_{3}-x_{2}\right\| \\
& +\left(C_{2} C_{1}+C_{1}+1\right)\left\|x_{1}-x_{0}\right\| \\
\leq & \left(\frac{1-C(\rho)^{i-1}}{1-C(\rho)} C_{2} C_{1}+C_{1}+1\right)\left\|x_{1}-x_{0}\right\| \\
< & \left(\frac{C_{1} C_{2}}{1-C(\rho)}+C_{1}+1\right) \eta \leq \rho, \\
\left\|x_{i+j}-x_{i}\right\| \leq & \left\|x_{i+j}-x_{i+j-1}\right\|+\left\|x_{i+j-1}-x_{i+j-2}\right\|+\ldots+\left\|x_{i+1}-x_{i}\right\| \\
\leq \quad & \left(C(\rho)^{i+j-3}+\ldots+C(\rho)^{i-2}\right)\left\|x_{3}-x_{2}\right\| \\
\leq \quad & C(\rho)^{i-2} \frac{1-C(\rho)^{j-1}}{1-C(\rho)}\left\|x_{3}-x_{2}\right\| \\
\leq C & C(\rho)^{i-2} \frac{1-C(\rho)^{j-1}}{1-C(\rho)} C_{2} C_{1}\left\|x_{1}-x_{0}\right\| \\
\leq \quad & C(\rho)^{i-2} \frac{1-C(\rho)^{j-1}}{1-C(\rho)} C_{2} C_{1} \eta .
\end{array}
$$


It follows from (23) that $x_{i} \in U\left(x_{0}, \rho\right)$; and from (24) that sequence $x_{i}$ is complete in a Banach space $X$. In particular, it converges to some $x^{*} \in \bar{U}\left(x_{0}, \rho\right)$. By letting $i \rightarrow+\infty$ in the estimate

$$
\begin{gathered}
\left\|B^{-1}\left(F_{1}\left(x_{i}\right)+F_{2}\left(x_{i}\right)\right)\right\|=\left\|B^{-1}\left(F_{1}\left(x_{i}\right)+F_{2}\left(x_{i}\right)-F_{1}\left(x_{i-1}\right)-F_{2}\left(x_{i-1}\right)-L_{i-2, i-1}\left(x_{i}-x_{i-1}\right)\right)\right\| \\
\leq \frac{w\left(\left\|x_{i-2}-x_{0}\right\|,\left\|x_{i}-x_{0}\right\|,\left\|x_{i-1}-x_{0}\right\|\right)\left\|x_{i}-x_{i-1}\right\|}{1-w_{0}\left(\left\|x_{i-1}-x_{0}\right\|,\left\|x_{i}-x_{0}\right\|\right)} \leq \frac{w(\rho, \rho, \rho)}{1-w_{0}(\rho, \rho)}\left\|x_{i}-x_{i-1}\right\|,
\end{gathered}
$$

we obtain $F_{1}\left(x^{*}\right)+F_{2}\left(x^{*}\right)=0$. The uniqueness part is omitted as analogous to the one in the local convergence case.

Hence, we can present our semilocal convergence result associated to the method (2).

Theorem 2. Assume that the conditions $(H)$ hold. Then, sequence $x_{k}$, defined by the method (2) for $x_{-1}, x_{0} \in D$, is well defined in $U\left(x_{0}, \rho\right)$; remains in $U\left(x_{0}, \rho\right)$; and converges to a solution $x^{*} \in \bar{U}\left(x_{0}, \rho\right)$ of Equation (1). On the other hand, the vector $x^{*}$ is the only solution of Equation (1) in $\bar{U}\left(x_{0}, \rho\right)$.

The same comments given in the previous remark hold.

\section{Numerical Experiment}

Consider the nondifferentiable system of equations

$$
\left\{\begin{array}{l}
3 x_{1}^{2} x_{2}+x_{2}^{2}-1+\left|x_{1}-1\right|^{3 / 2}=0, \\
x_{1}^{4}+x_{1} x_{2}^{3}-1+\left|x_{2}\right|^{3 / 2}=0 .
\end{array}\right.
$$

We therefore have an operator $F: \mathbb{R}^{2} \rightarrow \mathbb{R}^{2}$ such that $F=F_{1}+F_{2}$, as in (1), with $F_{1}, F_{2}: \mathbb{R}^{2} \rightarrow \mathbb{R}^{2}$, $F_{1}=\left(F_{1}{ }^{1}, F_{1}^{2}\right), F_{2}=\left(F_{2}{ }^{1}, F_{2}^{2}\right)$, being

$$
\begin{gathered}
F_{1}^{1}\left(x_{1}, x_{2}\right)=3 x_{1}^{2} x_{2}+x_{2}^{2}-1 \text { and } F_{1}^{2}\left(x_{1}, x_{2}\right)=x_{1}^{4}+x_{1} x_{2}^{3}-1, \\
F_{2}^{1}\left(x_{1}, x_{2}\right)=\left|x_{1}-1\right|^{3 / 2} \text { and } F_{2}^{2}\left(x_{1}, x_{2}\right)=\left|x_{2}\right|^{3 / 2},
\end{gathered}
$$

where the operator $F_{1}$ is continuously Fréchet-differentiable and $F_{2}$ is continuous but is a Fréchet nondifferentiable operator.

For $\mathbf{u}=\left(u_{1}, u_{2}^{T}\right), \mathbf{v}=\left(v_{1}, v_{2}\right)^{T} \in \mathbb{R}^{2}$, we consider the divided difference of first order defined by $[\mathbf{u}, \mathbf{v} ; F]=\left([\mathbf{u}, \mathbf{v} ; F]_{i j}\right)_{i, j=1}^{2} \in \mathcal{L}\left(\mathbb{R}^{2}, \mathbb{R}^{2}\right)$, where

$$
\begin{aligned}
& {[\mathbf{u}, \mathbf{v} ; F]_{i 1}=\left\{\begin{array}{l}
\frac{F_{i}\left(u_{1}, u_{2}, \ldots, v_{m}\right)-F_{i}\left(u_{1}, v_{2}, \ldots, v_{m}\right)}{u_{1}-v_{1}}, \text { if } u_{2} \neq v_{2}, \\
0, \text { if } u_{1}=v_{1},
\end{array}\right.} \\
& {[\mathbf{u}, \mathbf{v} ; F]_{i 2}=\left\{\begin{array}{l}
\frac{F_{i}\left(u_{1}, u_{2}, \ldots, v_{m}\right)-F_{i}\left(u_{1}, v_{2}, \ldots, v_{m}\right)}{u_{2}-v_{2}}, \text { if } u_{2} \neq v_{2}, \\
0, \text { if } u_{2}=v_{2},
\end{array}\right.}
\end{aligned}
$$

for $i=1,2$.

The iterative processes given by (1) allow us to consider direct iterative processes, such as (5) and (6); as well as iterative processes that use the decomposition method, such as (7) and (8). In this experiment, for the nondifferentiable system (25), we check that the application of the iterative processes that use the decomposition method have better behavior than the direct methods. 
To carry out this study, we will consider as an approximate solution of system (25):

$$
\mathbf{x}^{*}=(0.9383410452297656,0.3312445136375143),
$$

the starting points $\mathbf{x}_{-\mathbf{1}}=(5,5)$ and $\mathbf{x}_{\mathbf{0}}=(1,0)$, and use a tolerance $\left\|\mathbf{x}_{\mathbf{n}+\mathbf{1}}-\mathbf{x}_{\mathbf{n}}\right\| \leq 10^{-16}$. In these conditions, in Tables 1 and 2 we can see the results of the application of the direct iterative processes, the secant-type, and Kurchatov methods. Whereas in Tables 3 and 4 we can see the results of the application of the iterative processes that use the decomposition method, Newton-secant-type and Newton-Kuchatov methods. Observing the results obtained, it is evident that the best behavior of the iterative processes is given by (2) using the decomposition method.

Table 1. $\left\|\mathbf{x}^{*}-\mathbf{x}_{\mathbf{n}}\right\|$ for secant-type methods (5) and different values of the parameter $\lambda$.

\begin{tabular}{cccc}
\hline $\boldsymbol{n}$ & $\lambda=\mathbf{0}$ & $\lambda=\mathbf{0 . 5}$ & $\lambda=\mathbf{0 . 9 9}$ \\
\hline 1 & $3.18484 \times 10^{-1}$ & $2.965 \times 10^{-1}$ & $4.54388 \times 10^{-2}$ \\
2 & $5.21264 \times 10^{-2}$ & $4.13083 \times 10^{-2}$ & $3.74494 \times 10^{-3}$ \\
3 & $3.66108 \times 10^{-3}$ & $2.35344 \times 10^{-3}$ & $2.94716 \times 10^{-5}$ \\
4 & $2.59348 \times 10^{-4}$ & $7.2935 \times 10^{-5}$ & $3.69966 \times 10^{-9}$ \\
5 & $1.30031 \times 10^{-6}$ & $1.24012 \times 10^{-7}$ & $2.10942 \times 10^{-15}$ \\
6 & $4.42187 \times 10^{-10}$ & $6.07747 \times 10^{-12}$ & \\
7 & $1.11022 \times 10^{-15}$ & $1.11022 \times 10^{-16}$ & \\
\hline
\end{tabular}

Table 2. Kurchatov method (6).

\begin{tabular}{lc}
\hline $\boldsymbol{n}$ & $\left\|\mathbf{x}^{*}-\mathbf{x}_{\mathbf{n}}\right\|$ \\
\hline 1 & $2.99754 \times 10^{-1}$ \\
2 & $1.07269 \times 10^{-1}$ \\
3 & $4.20963 \times 10^{-2}$ \\
4 & $8.37098 \times 10^{-3}$ \\
5 & $2.78931 \times 10^{-4}$ \\
6 & $9.0784 \times 10^{-8}$ \\
7 & $2.9826 \times 10^{-11}$ \\
\hline
\end{tabular}

Table 3. $\left\|\mathbf{x}^{*}-\mathbf{x}_{\mathbf{n}}\right\|$ for Newton-secant-type methods (7) and different values of the parameter $\lambda$.

\begin{tabular}{cccc}
\hline $\boldsymbol{n}$ & $\lambda=\mathbf{0}$ & $\lambda=\mathbf{0 . 5}$ & $\lambda=\mathbf{0 . 9 9}$ \\
\hline 1 & $2.3538 \times 10^{-1}$ & $1.00278 \times 10^{-1}$ & $4.29554 \times 10^{-2}$ \\
2 & $3.48717 \times 10^{-1}$ & $2.88094 \times 10^{-2}$ & $2.53626 \times 10^{-3}$ \\
3 & $1.47537 \times 10^{-1}$ & $1.90518 \times 10^{-3}$ & $9.06208 \times 10^{-6}$ \\
4 & $3.4371 \times 10^{-2}$ & $8.39107 \times 10^{-6}$ & $1.9925 \times 10^{-10}$ \\
5 & $3.08399 \times 10^{-3}$ & $4.78016 \times 10^{-9}$ & \\
6 & $4.63665 \times 10^{-5}$ & $7.38298 \times 10^{-15}$ & \\
7 & $4.05776 \times 10^{-8}$ & & \\
8 & $2.96929 \times 10^{-13}$ & & \\
\hline
\end{tabular}

Table 4. Newton-Kurchatov method (8).

\begin{tabular}{cc}
\hline $\boldsymbol{n}$ & $\left\|\overline{\boldsymbol{x}^{*}}-\overline{x_{n}}\right\|$ \\
\hline 1 & $6.1659 \times 10^{-1}$ \\
2 & $4.75269 \times 10^{-3}$ \\
3 & $6.29174 \times 10^{-5}$ \\
4 & $3.37027 \times 10^{-9}$ \\
\hline
\end{tabular}


Remark 2. In the above example, we have selected the initial guess in a region where the operator is not smooth. The methods can be applied to systems where the operator is not smooth at the solution.

For instance, for the system:

$$
\left\{\begin{array}{l}
\left|x_{1}^{2}-1\right|+x_{2}-1=0 \\
x_{1}+x_{2}^{2}-1=0
\end{array}\right.
$$

the solution is $(1,1)$. If we take as initial guess $(0.5,0.5)$, the Steffensen method gives as errors $3.12 \times 10^{-2}$, $5.48 \times 10^{-4}, 1.92 \times 10^{-7}, 2.15 \times 10^{-14}$, we observe its second order.

\section{Boundary Value Problem: Discretization via the Multiple Shooting Method}

We will use the multiple shooting method for the discretization of boundary problems of the type

$$
y^{\prime \prime}(t)=f\left(t, y(t), y^{\prime}(t)\right), \quad y(a)=\alpha, \quad y(b)=\beta
$$

Thus, we should find the solution of the following nonlinear system of equations $F(s)=0$, where $F: \mathbb{R}^{N} \longrightarrow \mathbb{R}^{N}$ and

$$
\left\{\begin{aligned}
F_{1}\left(s_{0}, s_{1}, \ldots, s_{N-1}\right) & =s_{1}-y^{\prime}\left(t_{1} ; s_{0}\right) \\
F_{2}\left(s_{0}, s_{1}, \ldots, s_{N-1}\right) & =s_{2}-y^{\prime}\left(t_{2} ; s_{0}, s_{1}\right) \\
& \vdots \\
F_{N-1}\left(s_{0}, s_{1}, \ldots, s_{N-1}\right) & =s_{N-1}-y^{\prime}\left(t_{N-1} ; s_{0}, s_{1}, \ldots, s_{N-2}\right) \\
F_{N}\left(s_{0}, s_{1}, \ldots, s_{N-1}\right) & =\beta-y\left(t_{N} ; s_{0}, s_{1}, s_{N-2}, s_{N-1}\right) .
\end{aligned}\right.
$$

for a discretization of $[a, b]$ with $N$ subintervals,

$$
\text { TN } j, \quad T=b-a, \quad j=0,1, \ldots, N
$$

We consider the secant-type method

$$
\left\{\begin{array}{l}
\text { Given } y_{-1}, y_{0} \in D, \\
z_{n}=\lambda_{n} y_{n}+\left(1-\lambda_{n}\right) y_{n-1}, \quad \lambda_{n} \in[0,1), \\
y_{n+1}=y_{n}-\left[z_{n}, y_{n} ; F\right]^{-1} F\left(y_{n}\right), \quad n \geq 0,
\end{array}\right.
$$

where $\lambda_{n}$ is such that $\left\|z_{n}-x_{n}\right\| \leq$ Tol for a given tolerance, and Newton's method

$$
\left\{\begin{array}{l}
\text { Given } y_{0} \in D, \\
y_{n+1}=y_{n}-F\left(y_{n}\right)^{-1} F\left(y_{n}\right), \quad n \geq 0 .
\end{array}\right.
$$

We perform a numerical comparison between both methods. As we can see, in the multiple shooting method, the iterative schemes are used as black boxes. 
For the initial slope $\vec{s}_{0}=\left(s_{0}^{0}, s_{1}^{0}, \ldots, s_{N-1}^{0}\right)$, we propose

$$
\left\{\begin{aligned}
s_{0}^{0} & =\frac{\beta-\alpha}{b-a}=\frac{y\left(t_{N}\right)-y\left(t_{0}\right)}{t_{N}-t_{0}} \\
s_{1}^{0} & =\frac{y\left(t_{N}\right)-y\left(t_{1} ; s_{0}\right)}{t_{N}-t_{1}} \\
s_{2}^{0} & =\frac{y\left(t_{N}\right)-y\left(t_{2} ; s_{0}, s_{1}\right)}{t_{N}-t_{2}} \\
& \vdots \\
s_{N-1}^{0} & =\frac{y\left(t_{N}\right)-y\left(t_{N-1} ; s_{0}, s_{1}, \ldots, s_{N-2}\right)}{t_{N}-t_{N-1}} .
\end{aligned}\right.
$$

We analyze this particular example ([21], p. 554):

$$
\begin{aligned}
& y^{\prime \prime}(t)=\tau \cdot \sinh (\tau \cdot y(t)), \\
& y(0)=0, \quad y(1)=1 .
\end{aligned}
$$

We take $\tau=2.5$ and $N=4$ subintervals.

This problem admits the solution:

$$
y(t)=\frac{2}{\tau} \arg \sinh \left(\frac{s}{2} \cdot \frac{\operatorname{sn}\left(\tau t, 1-s^{2} / 4\right)}{\operatorname{cn}\left(\tau t, 1-s^{2} / 4\right)}\right),
$$

where

$$
s=y^{\prime}(0)=0.3713363932677645
$$

and $\operatorname{sn}(\cdot, \cdot)$ and $\operatorname{cn}(\cdot, \cdot)$ are the Jacobi elliptic functions.

Newton's method (29),

\begin{tabular}{cccc}
\hline$n$ & $\left\|F\left(\vec{s}_{n}\right)\right\|_{\infty}$ & $\left\|y(t)-y_{n}\right\|_{\infty}$ & $\left\|y^{\prime}(t)-y_{n}^{\prime}\right\|_{\infty}$ \\
\hline 0 & $10^{0}$ & $10^{-1}$ & $10^{0}$ \\
\hline 1 & $10^{-1}$ & $10^{-1}$ & $10^{-1}$ \\
\hline 2 & $10^{-2}$ & $10^{-2}$ & $10^{-2}$ \\
\hline 3 & $10^{-4}$ & $10^{-4}$ & $10^{-4}$ \\
\hline 4 & $10^{-7}$ & $10^{-7}$ & $10^{-7}$ \\
\hline 5 & $10^{-15}$ & $10^{-15}$ & $10^{-14}$ \\
\hline
\end{tabular}

Secant-type method (28),

\begin{tabular}{ccccc}
\hline$n$ & $\left\|F\left(\vec{s}_{n}\right)\right\|_{\infty}$ & $\left\|y(t)-y_{n}\right\|_{\infty}$ & $\left\|y^{\prime}(t)-y_{n}^{\prime}\right\|_{\infty}$ & $\left\|F^{\prime}\left(y_{n}\right)-\left[y_{n}, x_{n} ; F\right]\right\|_{\infty}$ \\
\hline 0 & $10^{0}$ & $10^{-1}$ & $10^{0}$ & $10^{-6}$ \\
\hline 1 & $10^{-1}$ & $10^{-1}$ & $10^{-1}$ & $10^{-6}$ \\
\hline 2 & $10^{-2}$ & $10^{-2}$ & $10^{-2}$ & $10^{-7}$ \\
\hline 3 & $10^{-4}$ & $10^{-4}$ & $10^{-4}$ & $10^{-6}$ \\
\hline 4 & $10^{-7}$ & $10^{-7}$ & $10^{-7}$ & $10^{-6}$ \\
\hline 5 & $10^{-15}$ & $10^{-15}$ & $10^{-14}$ & $10^{-6}$ \\
\hline
\end{tabular}


The methods using Jacobians obtain their order of convergence. However, in this example, the computation of the derivatives involves the approximation of a more complicated problem. For this reason, the methods free of derivatives are preferred, see [21]. Of course, we need to compute a good approximation to the Jacobian, this is the motivation of our parameters $\lambda_{n}$. For more similar examples and conclusions, we refer [22].

Remark 3. In many cases, when we manipulate an image, some random noise appears. This noise makes the later steps of processing the image difficult and inaccurate.

Let $f: \Omega \rightarrow \mathbb{R}$ be a noise signal or image.

Introducing the variable $w$ :

$$
w=\frac{\nabla u}{\sqrt{|\nabla u|^{2}}},
$$

the Total-Variation model is equivalent to the nonlinear and nondifferentiable system:

$$
\begin{aligned}
-\nabla \cdot w+\lambda(u-f) & =0, \\
w \sqrt{|\nabla u|^{2}}-\nabla u & =0 .
\end{aligned}
$$

This system should be discretized using finite differences and the associated nonlinear system of equations can be approximated by our family (see [23] for more details).

\section{Conclusions}

This paper was devoted to the analysis of a general family of two-point Newton-type methods for smooth and nonsmooth operators. We have considered two types of convergence-semilocal and local. The family includes a great number of methods. We have applied the schemes to several interesting problems, in particular to nonsmooth nonlinear systems, boundary problems, and image denoising models.

Author Contributions: All the authors have been contributed similarly and they are participated in all the work. Funding: This research received no external funding.

Acknowledgments: Research of the first and third authors supported in part by Programa de Apoyo a la investigación de la fundación Séneca-Agencia de Ciencia y Tecnología de la Región de Murcia 20928/PI/18 and by MTM2015-64382-P. Research of the fourth and fifth authors supported by Ministerio de Economía y Competitividad under grant MTM2014-52016-C2-1P.

Conflicts of Interest: The authors declare no conflict of interest.

\section{References}

1. Amat, S.; Busquier, S. A modified secant method for semismooth equations. Appl. Math. Lett. 2003, 16, 877-881. [CrossRef]

2. Amat, S.; Busquier, S. On a higher order secant method. Appl. Math. Comput. 2003, 141, 321-329. [CrossRef]

3. Amat, S.; Busquier, S. A two-step Steffensen's method under modified convergence conditions. J. Math. Anal. Appl. 2006, 324, 1084-1092. [CrossRef]

4. Argyros, I.K.; Magreñán, A.A. Iterative Methods and Their Dynamics with Applications: A Contemporary Study; CRC Press: Boca Raton, FL, USA, 2017.

5. Argyros, I.K.; Magreñán, A.A. A Contemporary Study of Iterative Methods; Academic Press: Cambridge, MA, USA, 2018.

6. Grau-Sánchez, M. Noguera, M.; Amat, S. On the approximation of derivatives using divided difference operators preserving the local convergence order of iterative methods. J. Comput. Appl. Math. 2013, 237, 363-372.

7. Amat, S.; Bermúdez, C.; Busquier, S.; Mestiri, D. A family of Halley-Chebyshev iterative schemes for non-Fréchet differentiable operators. J. Comput. Appl. Math. 2009, 228, 486-493. [CrossRef] 
8. Barton̆, M. Solving polynomial systems using no-root elimination blending schemes. Comput.-Aided Des. 2011, 43, 1870-1878. [CrossRef]

9. Barton̆, M.; Elber, G.; Hanniel, I. Topologically guaranteed univariate solutions of underconstrained polynomial systems via no-loop and single-component tests. Comput.-Aided Des. 2011, 43, 1035-1044. [CrossRef]

10. Hanniel, I.; Elber, G. Subdivision termination criteria in subdivision multivariate solvers using dual hyperplanes representations. Comput.-Aided Des. 2007, 39, 369-378. [CrossRef]

11. Chen, J.; Shen, Z. Convergence analysis of the secant type methods. Appl. Math. Comput. 2007, 188, 514-524. [CrossRef]

12. Hongmin, R.; Qingiao, W. The convergence ball of the Secant method under Hölder continuous divided differences. J. Comput. Appl. Math. 2006, 194, 284-293. [CrossRef]

13. Kewei, L. Homocentric convergence ball of the Secant method. Appl. Math. J. Chin. Univ. Ser. B 2007, 22, 353-365.

14. Ren, H.; Argyros, I.K. Local convergence of efficient Secant-type methods for solving nonlinear equations. Appl. Math. Comput. 2012, 218, 7655-7664. [CrossRef]

15. Zincenko, A.I. Some Approximate Methods of Solving Equations with Non-Differentiable Operators; Dopovidi Akad Nauk: 1963; pp. 156-161.

16. Hernández, M.A.; Rubio, M.J. A uniparametric family of iterative processes for solving nondifferentiable equations. J. Math. Anal. Appl. 2002, 275, 821-834. [CrossRef]

17. Hernández, M.A.; Rubio, M.J.; Ezquerro, J.A. Secant-like methods for solving nonlinear integral equations of the Hammerstein type. J. Comput. Appl. Math. 2000, 115, 245-254. [CrossRef]

18. Kurchatov, V.A. On a method of linear interpolation for the solution of funcional equations. Dolk. Akad. Nauk SSSR 1971, 198, 524-526; translation in Soviet Math. Dolk. 1971, 12, 835-838. (In Russian)

19. Hernández, M.A.; Rubio, M.J. On a Newton-Kurchatov-type Iterative Process. Numer. Funct. Anal. 2016, 37, 65-79. [CrossRef]

20. Kantorovich, L.V.; Akilov, G.P. Functional Analysis; Pergamon Press: Oxford, UK, 1982.

21. Stoer, J.; Bulirsch, R. Introduction to Numerical Analysis, 2nd ed.; Springer: New York, NY, USA, 1993.

22. Alarcón, V.; Amat, S.; Busquier, S.; López, D.J. A Steffensen's type method in Banach spaces with applications on boundary value problems. J. Comput. Appl. Math. 2008, 216, 243-250. [CrossRef]

23. Amat, S.; Argyros, I.K.; Busquier, S.; Hernández-Verón, M.A.; Martínez, E. A unified convergence analysis for single step-type methods for non-smooth operators. 2019, submitted.

(c) 2019 by the authors. Licensee MDPI, Basel, Switzerland. This article is an open access article distributed under the terms and conditions of the Creative Commons Attribution (CC BY) license (http:/ / creativecommons.org/licenses/by/4.0/). 